\title{
The concept of vibration damping of the variable mass assembly
}

\author{
Rafat Kwiatkowski ${ }^{1 *}$ \\ ${ }^{1}$ The President Stanisław Wojciechowski State University of Applied Sciences in Kalisz; Nowy Świat 4, \\ 62-800 Kalisz, Poland
}

\begin{abstract}
An innovative method of damping vibrations using a variable mass assembly will be presented in this article. The concept was based on a physical pendulum model consisting of two connected elements. Mass exchange between the upper and lower member of the pendulum is modelled as a gravity a fluid flow. With the passage of time, the mass of the upper member of pendulum decreases and the mass of the lower member of pendulum increases. It is assumed preserve the mass of the whole system. The article will also present the application possibilities and a review of currently used vibration dampers.
\end{abstract}

Keywords: vibration damping, variable mass assembly, physical pendulum model

\section{Introduction}

In the construction and operation of machines, the phenomenon of mechanical vibration is analyzed in three aspects:

- impact on machine quality (sensitivity class, described in PN-85/B-02170),

- approaching machines to emergency conditions (vibroacoustic diagnostics),

- impact on quality of work and health of operators (vibroacoustic protection).

Nowadays, each of the above aspects has significant achievements described in very extensive literature among others in numerous works of Prof. Czesław Cempel. The interest of the systems with variable mass emerged at the time during the search for the effects of physical (mechanical), possible to use in the construction of vibration dampers. These were mostly experimental studies without much success, as evidenced by the level of available products on the market. Due to the patent protection, also lacks bibliography of these studies. In such a situation arose to wait for theoretical modelling of dynamic systems with variable mass.

\footnotetext{
*Corresponding author: kwiatkowski-rafal@o2.pl

Reviewers: Michat Bartyś, Milan Vaško
} 


\section{Currently used vibration dampers}

Due to the growing need and trend for the design of engineering structures with greater durability and care for improving the comfort of their users (reduction of vibrations, minimization of external forces effects, including wind, seismic and paraseismic effects), various types of vibration eliminators are increasingly used, devices installed on a structure or forming part of a structure whose task is to minimize the effects of dynamic forces on the structure.

\subsection{Passive control systems}

Passive control systems are the most commonly used vibration eliminators. These are relatively technically simple devices or structural elements, built in such a way that the construction as a whole has great potential for dissipating the energy provided by the forcing forces. An important part of passive vibration eliminators are the so-called dynamic vibration dampers. They modify the natural frequency of the structure and in this way contribute to reducing the effects of dynamic loads. An important feature of passive vibration eliminators is that their operation isn't related to the need to supply the silencer with an external energy source. Another feature of passive vibration eliminators is the stability of the parameters of these silencers (eg. viscosity coefficient). These parameters can't be intentionally modified during the silencer operation [1].

Among the passive control systems can be mentioned:

- visco-elastic vibration dampers - on a large scale, they were used for the first time in World Trade Center skyscrapers (10,000 pieces in each tower). These silencers use the dissipation effect of energy in materials with visco-elastic properties placed between the steel elements of the structure.

- metal vibration eliminators - used most often as a protection of buildings against the effects of earthquakes. The dissipation of energy is the result of plastic deformation of plates made of mild steel.

- mass (dynamic) vibration dampers - they are used to reduce vibrations caused by wind and earthquakes. They contain an additional mass connected to the structure by means of a spring element and an energy dissipation element.

\subsection{Active vibration eliminators}

The essence of active vibration regulation boils down to the idea of generating additional forces acting on the structure so that they counteract dynamic loads. The active vibration eliminator is essentially an automatic adjustment system mounted on the structure. The main elements of this system are sensors, a computer and actuators. The sensors are located at various points of the structure and measure its dynamic state. This information is sent to the computer, which on this basis determines the desired values of the control force. Then signals are sent to the actuators, which, in turn, trigger the appropriate control forces and act on the structure. The most important advantages of active vibration eliminators include: the possibility of significant reduction of vibration amplitudes and accelerations of the structure. Disadvantages: devices requiring high active regulation forces are necessary and therefore there is a high demand for energy [1]. 


\subsection{Quasi-active vibration dampers}

Semi-active vibration dampers combine the beneficial properties of both passive and active vibration eliminators. Similarly to active systems, they are built of sensors, computer and actuators, but they do not require such a high power supply to reduce vibrations, which is extremely important and beneficial. Often, quasi-active silencers can work effectively using only battery power. This is extremely important in emergencies, when external power sources may turn out to be unreliable. Application example: Kajima Shizuoka building in Japan [2].

\subsection{Hybrid vibration dampers}

The hybrid silencer is a combination of a mass silencer with an additional active element, whose task is to improve the efficiency of the passive solution. The efficiency of such a silencer depends mainly on the proper tuning of the mass silencer. The forces from the active actuator increase its effectiveness and ensure its effective operation in the event of possible change of the dynamic characteristics of the structure during its operation. Application example: Landmark Tower building in Yokohama [2].

\section{The concept of damping vibrations by means of a double- pendulum system with variable mass}

In technical sciences, apart from thermodynamics, mainly so-called isolated systems that do not interact with the environment are considered. In mechanics, systems composed of subsystems most often interact with each other mainly with the participation of forces and force pairs (moments of forces). The simplest mechanical model of interacting subsystems is the so-called double pendulum. Literature [3] describes many examples of spectacular exchange of kinetic energy between the members of different vibrating systems. These are systems and subsystems with a constant mass.

The purpose of this paper is an attempt to describe the mathematical phenomenon of mass exchange between the members of the double pendulum and its impact on the dynamics of the whole system.

According to tradition, already Isaac Newton, writing the famous dynamic equation, in the form:

$$
\frac{d}{d t}(m \vec{v})=\vec{F}
$$

suggested that the mass of the mechanical system could change over time.

This has led to the use of the term "variable mass" in the history of mechanics in three cases:

- change of mass distribution in a fixed mass system,

- change the mass of the system while maintaining its volume,

- changes in the mass of the system and its volume.

It's easy to give examples of these cases. The first one occurs during the movement of multiple bodies when their center of mass changes position over time. The second occurs when the fluid leaks from the tank under the influence of gravity. The third occurs when we investigate the motion of the evaporation drop.

The above description can be given a quantitative description. Let us assume that the mass of the system $m[\mathrm{~kg}]$ is a continuous, differential function of two variables: density $\rho\left[\mathrm{kg} / \mathrm{m}^{3}\right]$ and volume $V\left[\mathrm{~m}^{3}\right]$, which in general may depend on spatial coordinates and time: 


$$
m=m(\rho, V) .
$$

The total differential of the mass has the form:

$$
d m=\left(\frac{\partial m}{\partial \rho}\right)_{V} d \rho+\left(\frac{\partial m}{\partial V}\right)_{\rho} d V
$$

Differential variables must have their physical dimensions:

$$
d \rho\left[\frac{\mathrm{kg}}{\mathrm{m}^{3}}\right], \quad d V\left[\mathrm{~m}^{3}\right] .
$$

This facilitates the interpretation of partial derivatives:

$$
\begin{gathered}
\left(\frac{\partial m}{\partial \rho}\right)_{V}\left[m^{3}\right]-\text { as volume, } \\
\left(\frac{\partial m}{\partial V}\right)_{\rho}\left[\frac{\mathrm{kg}}{\mathrm{m}^{3}}\right]-\text { as a mass density. }
\end{gathered}
$$

So we get:

$$
d m=V d p+\rho d V
$$

The above pattern allows to interpret the three preceding cases:

1. $d m=0 ; m=$ const. - describes the constancy of mass.

2. $d V=0 ; V=$ const. $; d m=V d \rho ; \frac{d m}{d t}=V \frac{d \rho}{d t}-$ describes the mass change at constant volume.

3. $d \rho=0 ; \rho=$ const. $; d m=\rho d V ; \frac{d m}{d t}=\rho \frac{d V}{d t}-\mathrm{d}$ escribes the mass change when changing volume.

Conducted research [4-9] include the study of the motion of a double pendulum subjected to variable initial conditions. The effects of initial deflections and initial velocities given separately to the system's members were analyzed. It is assumed that the total mass of a double pendulum is constant, however there is a mass flow between the members.

The practical implementation of the double pendulum is made of two hollow rods articulately connected to each other in the field of gravity. Additionally, they are connected to the line conveying the liquid flowing by gravity from the upper to the lower member. In the mathematical layer, classical formalism of Lagrange's equations of analytic mechanics was used for a system with two degrees of freedom with smooth, holonomic constraints, using the force of gravity as a potential force. The change in mass has been modeled by the phenomenon of gravitational outflow of liquid from a tank with a hole. It is idealized that the movement of the member (tank) does not disturb the outflow. The described system is modeled as a physical pendulum in computational simulations. 


$$
\begin{gathered}
\ddot{\alpha} L^{2}\left(M_{0}+m_{0}-\frac{2}{3} M_{0} e^{-\lambda \mathrm{t}}\right)+\dot{\alpha} L^{2} \frac{2}{3}\left(\lambda M_{0} e^{-\lambda \mathrm{t}}\right)+ \\
+\frac{1}{2} \ddot{\beta}\left(M_{0}+m_{0}-M_{0} e^{-\lambda \mathrm{t}}\right) L l \cos (\alpha-\beta)+\frac{1}{2} \dot{\beta}\left(\lambda M_{0} e^{-\lambda \mathrm{t}}\right) L l \cos (\alpha-\beta)+ \\
+\frac{1}{2} \dot{\beta}^{2}\left(M_{0}+m_{0}-M_{0} e^{-\lambda \mathrm{t}}\right) L l \sin (\alpha-\beta)+ \\
+g L\left(M_{0}+m_{0}-\frac{1}{2} M_{0} e^{-\lambda \mathrm{t}}\right) \sin \alpha=0 \\
\ddot{\beta} \frac{1}{3}\left(M_{0}+m_{0}-M_{0} e^{-\lambda \mathrm{t}}\right) l^{2}+\dot{\beta} \frac{1}{3}\left(\lambda M_{0} e^{-\lambda \mathrm{t}}\right) l^{2}+ \\
+\frac{1}{2} \ddot{\alpha}\left(M_{0}+m_{0}-M_{0} e^{-\lambda \mathrm{t}}\right) L l \cos (\alpha-\beta)+\frac{1}{2} \dot{\alpha}\left(\lambda M_{0} e^{-\lambda \mathrm{t}}\right) L l \cos (\alpha-\beta)- \\
-\frac{1}{2} \dot{\alpha}^{2}\left(M_{0}+m_{0}-M_{0} e^{-\lambda \mathrm{t}}\right) L l \sin (\alpha-\beta)+\frac{1}{2}\left(M_{0}+m_{0}-M_{0} e^{-\lambda \mathrm{t}}\right) g l \sin \beta=0
\end{gathered}
$$

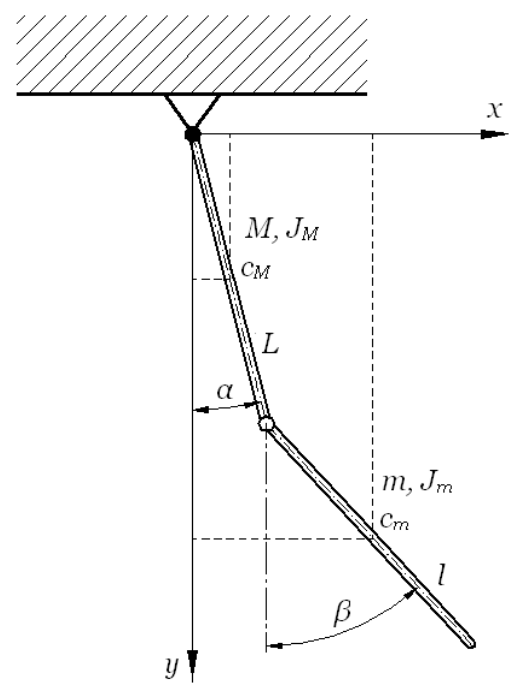

Fig. 1. Diagram of a double physical pendulum

In addition, the idealization is used that the center of gravity remains at the same point, which means that the masses throughout the whole body increase or decrease evenly over the entire length of the link. The Wolfram Research Mathematica program was used for numerical calculations and graphical presentation of results.

The work has included within the scope motion study of a double physical pendulum (Fig. 1) for three sets of initial conditions. These sets are different from each other only different, predetermined angle of the lower member of the pendulum $\beta$. Other initial conditions of the system, such as: $L$ - length of the upper, $l$ - length of the lower member of the pendulum, $M$ - mass of the upper, $m$ - mass of the lower member of the pendulum, $\alpha$ - angle of the upper member of the pendulum, $\alpha^{\prime}-$ generalized velocity of the upper, $\beta^{\prime}$ - generalized velocity of the lower member, $\lambda$ - coefficient of mass transfer, for both simulations are identical. It is assumed that the total mass of the double pendulum is constant, however the mass flow occurs between members.

The article is a continuation of the considerations discussed in the work of [4-9]. 


\section{Results}

Based on previous considerations described in the works [4-9], nonlinear equations for the double physical pendulum with variable mass are derived:

Results of scientific research presented in this paper, find to compare behavior and analyse chaotic systems, for nonlinear course. Due to the limited volume of this publication, only research results for a double pendulum with variable mass will be presented. Work on the dynamics of a double pendulum with a constant mass can be found in numerous publications, among others in [3]. The initial conditions shown in Table 1.

Table 1. The initial conditions

\begin{tabular}{|c|c|c|c|}
\hline \multicolumn{4}{|c|}{ Values of the pendulum parameters } \\
\hline $\mathbf{L}[\mathbf{m}]$ & $\mathbf{l}[\mathbf{m}]$ & & $\mathbf{m}_{0}[\mathrm{~kg}]$ \\
\hline 1 & 1 & & 0.10 \\
\hline \multicolumn{4}{|c|}{ Initial values for pendulums } \\
\hline$\alpha_{0}$ & $\beta_{0}$ & $\dot{\alpha}_{0}$ & $\lambda$ \\
\hline \multirow{3}{*}{0} & 0.10 & \multirow{3}{*}{0} & \multirow{3}{*}{0.015} \\
\hline & 0.25 & & \\
\hline & 0.20 & & \\
\hline
\end{tabular}

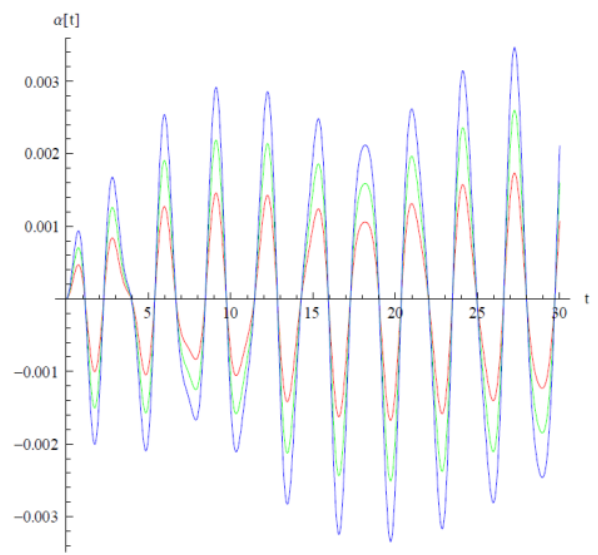

Fig. 2. Graph of the generalized coordinates of the $\alpha$ upper pendulum

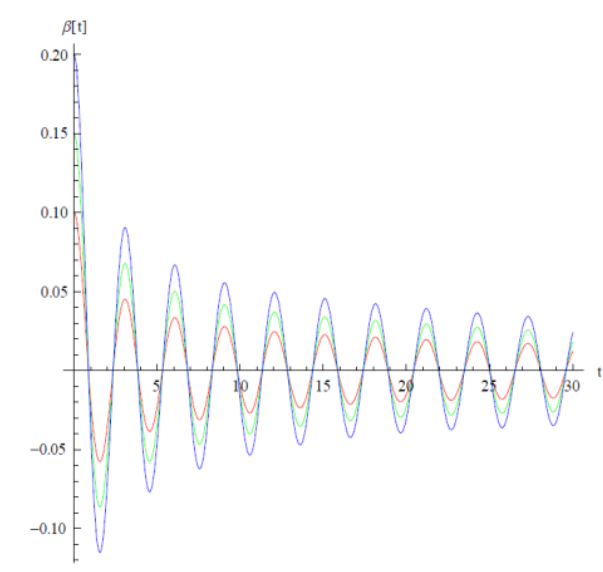

Fig. 3. Graph of generalized $\beta$ coordinates of the lower part of the pendulum 


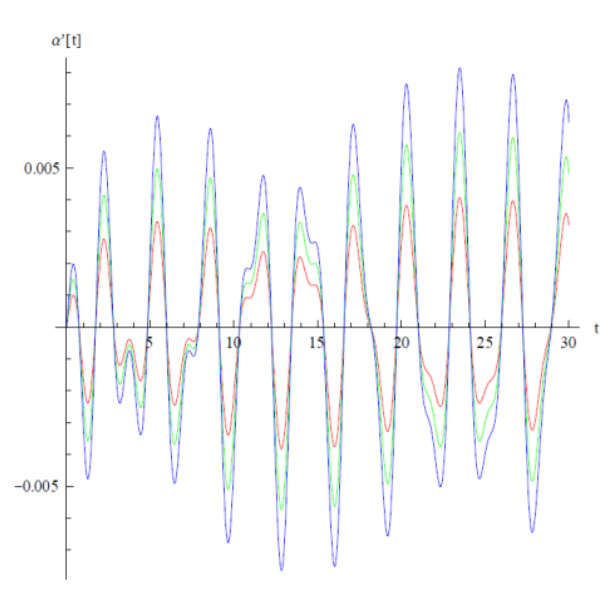

Fig. 4. Graph of the generalized top velocity of the pendulum

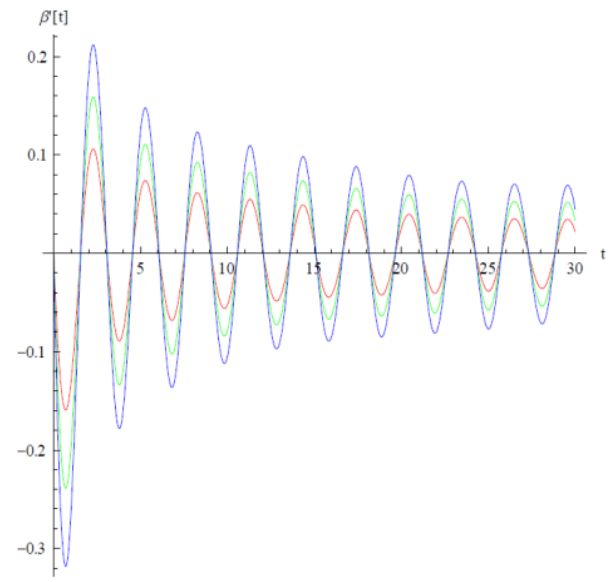

Fig. 5. Graph of the generalized speed of the lower part of the pendulum

For given initial parameters, values of deflection of the upper member of a double physical pendulum with variable mass (Fig. 2) for solutions of nonlinear equations increase with time for all three simulations, however their values are small, they reach a maximum of $0.016 \mathrm{rad}$ for the first, 0.025 advice for the second and 0.034 advice for the third simulation. The velocities values of the generalized upper pendulum members (Fig. 4) are also small and do not exceed $0.04 \mathrm{rad} / \mathrm{s}$ for the first, $0.006 \mathrm{rad} / \mathrm{s}$ for the second and $0.008 \mathrm{rad} / \mathrm{s}$ for the third simulation. Time progression of generalized coordinates $\beta$ (Fig. 3 ) and speeds of generalized $\beta^{\prime}$ (Fig. 5) of the lower member of the physical pendulum, show vibration damping. For all three simulations, the value of vibration amplitude decreases in the tested period of time from the initial set value of $0.1 \mathrm{rad}$, to $0.02 \mathrm{rad}$ for the first, from $0.15 \mathrm{rad}$ to $0.025 \mathrm{rad}$ for the second and from 0.2 rad to 0.03 advice for the third simulation. The values of the generalized speed of the lower part of the pendulum, for the three simulations, reach the maximum in the first phase of the process. For the first simulation $0.16 \mathrm{rad} / \mathrm{s}$, for the second simulation $0.24 \mathrm{rad} / \mathrm{s}$, and for the third simulation $0.32 \mathrm{rad} / \mathrm{s}$, after which, for all three simulations, the values of generalized velocities gradually decrease.

\section{Conclusions}

Simulations for a double physical pendulum with constant mass of both pendulum members, as to character, are consistent with the literature. The vibration energy is transferred (cyclically) between the members.

The most important conclusion from the research is that the vibration damping of the pendulum member can be reduced by increasing its mass.

As demonstrated, the solutions for the variable mass pendulum model differ significantly from those of the constant mass pendulum model. When the mass change in the system is relatively small compared to the total mass of the system, it is not necessary to include this fact in the study of the system's motion. On the other hand, in systems that are subject to significant mass changes, especially if this occurs in a short period of time, this process should be taken into account in order to study dynamics. Otherwise, any predicted system responses will be far from its true behavior [10].

These results open new research fields. The next stage of the research will be the preparation of an experimental stand which will enable the analysis of the mass transfer 
phenomenon in the real system and the comparison of the results obtained with the theoretical analysis.

\section{References}

1. R. Lewandowski, Reduction of vibrations in building structures. PWN, Warszawa (2014)

2. J. Kawecki, R. Masłowski, Use of passive, quasi-active and hybrid silencers to reduce seismic and paraseismic vibrations of buildings - overview of solutions. Czasopismo techniczne Civil Engineering, Wydawnictwo Politechniki Krakowskiej, 3-B/2010, 11, 10, 59-67 (2010)

3. D. Sado, The vibrations of regular and chaotic in the selected systems with pendulums. WNT, Warszawa (2010)

4. R. Kwiatkowski, Movement of double mathematical pendulum with variable mass. Machine Dynamics Research 38 (2), 47-58 (2014)

5. R. Kwiatkowski, Vibration Damping in the Double Mathematical Pendulum with Variable Mass. Machine Dynamics Research 38 (4), 23-32 (2014)

6. R. Kwiatkowski, Analysis of the dynamics of a double mathematical and physical pendulum with variable mass. Inżynieria Wytwarzania, Wydawnictwo uczelniane Państwowej Wyższej Szkoły Zawodowej im. Prezydenta Stanisława Wojciechowskiego w Kaliszu, 73-82, Kalisz (2014) ISBN 978 83-64090-41-7

7. R. Kwiatkowski, T.J. Hoffmann, A. Kołodziej, G. Domek, Dynamics of a double physical pendulum with variable mass in dimensionless coordinates. Mechanik 11/2016, 15841586 (2016)

8. R. Kwiatkowski, Dynamic analysis of double pendulum with variable mass and initial velocities, Procedia Engineering 136, 175-180 (2016)

9. M. Dudziak, R. Kwiatkowski, A. Kołodziej: The double pendulum as a variable mass system. International Conference „Methods \& Tools for CAE - concepts and applications", 267-273 (2017)

10. F.O. Eke, T.C. Mao, On the dynamics of variable mass systems. The International Journal of Mechanical Engineering Education 30(2), 123-137 (2002) 